J. Nonlinear Var. Anal. 2 (2018), No. 1, pp. 25-33

Available online at http://jnva.biemdas.com

https://doi.org/10.23952/jnva.2.2018.1.03

\title{
EXISTENCE AND UNIQUENESS OF SOLUTIONS FOR A NONLINEAR FRACTIONAL INITIAL VALUE PROBLEM INVOLVING CAPUTO DERIVATIVES
}

\author{
JÜRGEN APPELL ${ }^{1, *}$, BELÉN LÓPEZ² ${ }^{2}$ KISHIN SADARANGANI ${ }^{2}$ \\ ${ }^{1}$ Universität Würzburg, Mathematisches Institut, Campus Hubland Nord, Emil-Fischer-Str. 30, D-97074 Würzburg, Germany \\ ${ }^{2}$ Universidad de Las Palmas de Gran Canaria, Departamento de Matemáticas, \\ Campus de Tafira Baja, E-35017 Las Palmas de G.C., Spain
}

\begin{abstract}
In this paper, we give a sufficient condition for the existence and uniqueness interval of continuous solutions to a class of fractional initial value problems involving Caputo derivatives when the data functions satisfy a non-classical Lipschitz condition. The main tool used in the paper is a fixed point theorem for generalized contractions due to Browder-Matkowski.

Keywords. Initial value problem; Caputo derivative; Singular integral equation; Browder fixed point theorem; Matkowski fixed point theorem.
\end{abstract}

2010 Mathematics Subject Classification. 47H10, 34A12, 26 A33.

\section{INTRODUCTION}

The following result may be found in the book [4].

Theorem 1.1. Suppose that $f:[a, b] \times \mathbb{R} \rightarrow \mathbb{R}$ is continuous and satisfies a Lipschitz condition

$$
|f(t, x)-f(t, y)| \leq k|x-y| \quad(t \in[a, b], x, y \in \mathbb{R})
$$

with respect to the second variable. If $b-a<\frac{2 \sqrt{2}}{\sqrt{k}}$, then the boundary value problem

$$
\left\{\begin{array}{l}
y^{\prime \prime}(t)=-f(t, y(t)) \quad(a<t<b), \\
y(a)=A, y(b)=B
\end{array}\right.
$$

where $A, B \in \mathbb{R}$, has a unique continuous solution on $[a, b]$.

Of course, Theorem 1.1 has been generalized in many directions. For example, in the recent paper [3], the following more general result has been proved, where the second derivative in (1.2) is replaced by a fractional Riemann-Liouville derivative.

Theorem 1.2. Suppose that $f:[a, b] \times \mathbb{R} \rightarrow \mathbb{R}$ is continuous and satisfies (1.1). If

$$
b-a<\frac{\Gamma(\alpha)^{\frac{1}{\alpha}} \alpha^{\frac{\alpha+1}{\alpha}}}{k^{\frac{1}{\alpha}}(\alpha-1)^{\frac{\alpha-1}{\alpha}}},
$$

\footnotetext{
${ }^{*}$ Corresponding author.

E-mail addresses: jurgen@dmuw.de (J. Appell), belen.lopez@ulpgc.es (B. López), ksadaran@dma.ulpgc.es (K. Sadarangani).

Received May 31, 2017; Accepted August 25, 2017.
}

(c)2018 Journal of Nonlinear and Variational Analysis 
where $\Gamma(\alpha)$ is the Euler Gamma function, then the boundary value problem

$$
\left\{\begin{array}{l}
D^{\alpha} y(t)=-f(t, y(t)) \quad(a<t<b) \\
y(a)=0, y(b)=B
\end{array}\right.
$$

where $B \in \mathbb{R}, 1<\alpha \leq 2$, and $D^{\alpha}$ denotes the fractional Riemann-Liouville derivative of order $\alpha$, has a unique continuous solution on $[a, b]$.

Both theorems are proved by applying the classical Banach contraction mapping principle in the Banach space $C[a, b]$. Motivated by these results, in this paper we study the same question for the general fractional initial value problem

$$
\left\{\begin{array}{l}
D_{c}^{\alpha} x(t)=f(t, x(t))+D_{c}^{\alpha-1} g(t, x(t)) \quad(a<t<b), \\
x(a)=\theta_{1}, x^{\prime}(a)=\theta_{2}
\end{array}\right.
$$

where $1<\alpha<2, f, g:[a, b] \times \mathbb{R} \rightarrow \mathbb{R}$ are given continuous functions, $\theta_{1}, \theta_{2} \in \mathbb{R}$ and $D_{c}^{\alpha}$ denotes the Caputo fractional derivative of order $\alpha$. In what follows, we do not require that $f$ and $g$ satisfy the Lipschitz condition (1.1), but a more general condition. The main tool in our study is then a fixed point theorem due to Browder-Matkowski which we will recall in the following section.

To begin with, let us briefly recall the definition and some facts about fractional integration and differentiation. For more details, we refer the reader to the monographs $[5,8]$.

Definition 1.1. Let $\alpha \geq 0$ and $f$ be a real function defined on the interval $[a, b]$. The Riemann-Liouville fractional integral of order $\alpha$ of $f$ is defined for $t \in[a, b]$ by

$$
I^{\alpha} f(t)= \begin{cases}f(t), & \text { for } \alpha=0 \\ \frac{1}{\Gamma(\alpha)} \int_{a}^{t}(t-s)^{\alpha-1} f(s) d s, & \text { for } \alpha>0\end{cases}
$$

The operator $I^{\alpha}$ has many interesting properties. For instance, the semigroup property

$$
\left(I^{\alpha} \circ I^{\beta}\right) f(t)=I^{\alpha+\beta} f(t) \quad(\alpha, \beta \geq 0)
$$

holds for any $t \in[a, b]$ and $f \in L^{1}[a, b]$. Of course, the values $\alpha \in(0,1)$ are particularly interesting, because the integral in (1.5) becomes then weakly singular.

Definition 1.2. Let $\alpha \geq 0$ and $f$ be a real function defined on the interval $[a, b]$. The Caputo fractional derivative of order $\alpha$ of $f$ is defined by

$$
D_{c}^{\alpha} f(t)= \begin{cases}f(t), & \text { for } \alpha=0 \\ \frac{1}{\Gamma(n-\alpha)} \int_{a}^{t}(t-s)^{n-\alpha-1} f^{(n)}(s) d s, & \text { for } \alpha>0\end{cases}
$$

for any $t \in[a, b]$, where $n=[\alpha]+1$ and $[\alpha]$ denotes the integer part of $\alpha$, provided that the right side of (1.7) is pointwise defined.

An important connection between the Riemann-Liouville fractional integral and the Caputo fractional derivative reads as follows. 
Lemma 1.1. Suppose that $f \in C^{n-1}[a, b]$ and $f^{(n)}$ exists almost everywhere on $[a, b]$. Then

$$
\left(I^{\alpha} \circ D_{c}^{\alpha}\right) f(t)=f(t)-\sum_{k=0}^{n-1} \frac{f^{(k)}(a)}{k !}(t-a)^{k} .
$$

In particular, for $0<\alpha<1$ and $n=1$, we have

$$
\left(I^{\alpha} \circ D_{c}^{\alpha}\right) f(t)=f(t)-f(a),
$$

while for $1<\alpha<2$ and $n=2$ we have

$$
\left(I^{\alpha} \circ D_{c}^{\alpha}\right) f(t)=f(t)-f(a)-f^{\prime}(a)(t-a) .
$$

The equality (1.9) may be regarded as a fractional analogue to the Lagrange mean value theorem for differentiable functions.

\section{GenerALIZED CONTRACTIONS}

Now we state the fixed point theorem for generalized contractions which we will use for proving our main existence and uniqueness result for (1.4).

Theorem 2.1. Let $(X, d)$ be a complete metric space and $T: X \rightarrow X$ a mapping such that

$$
d(T x, T y) \leq \phi(d(x, y)) \quad(x, y \in X),
$$

where $\phi:[0, \infty) \rightarrow[0, \infty)$ is a nondecreasing function such that one of the following conditions holds.

(1) For any $t>0$ we have

$$
\lim _{n \rightarrow \infty} \phi^{(n)}(t)=0,
$$

where $\phi^{(n)}$ denotes the $n$-th iteration of $\phi$.

(2) The function $\phi$ is right-continuous and satisfies

$$
\phi(t)<t \quad(t>0) .
$$

Then $T$ has a unique fixed point in $X$.

Of course, the choice $\phi(t)=k t$ with $0<k<1$ gives the classical Banach contraction mapping principle. Functions $\phi$ which satisfy (1) or (2) are called comparison functions in the book [10], and we will also use this name in the sequel.

We remark that Theorem 2.1 was proved under the hypothesis (1) in [2], and under the hypothesis (2) in [6]. As a matter of fact, one may show that these two conditions are in a certain sense equivalent. Although this seems to be well known (see $[1,10]$ ) we give a short proof for the sake of completeness and the convenience of the reader.

Lemma 2.1. Condition (2.2) implies condition (2.3). Conversely, if $\phi$ is right-continuous, condition (2.3) implies condition (2.2).

Proof. Suppose first that (2.2) is true, but (2.3) is not. Then there exists $t_{0}>0$ such that $t_{0} \leq \phi\left(t_{0}\right)$. Since $\phi$ is nondecreasing, it follows that $\left(\phi^{(n)}\left(t_{0}\right)\right)_{n}$ is a nondecreasing sequence, and thus

$$
0<t_{0} \leq \lim _{n \rightarrow \infty} \phi^{(n)}\left(t_{0}\right)
$$


contradicting condition (1). Conversely, suppose now that $\phi$ is right-continuous and (2.3) is true, but (2.2) is not. Then we can find $t_{0}>0$ such that

$$
\limsup _{n \rightarrow \infty} \phi^{(n)}\left(t_{0}\right) \neq 0 \text {. }
$$

Since $\phi\left(t_{0}\right)<t_{0}$ and $\phi$ is nondecreasing, the sequence $\left(\phi^{(n)}\left(t_{0}\right)\right)_{n}$ is decreasing and nonnegative. Consequently, the limit

$$
a:=\lim _{n \rightarrow \infty} \phi^{(n)}\left(t_{0}\right)
$$

exists and is strictly positive. Using the right-continuity of $\phi$, we conclude that

$$
\phi(a)=\phi\left(\lim _{n \rightarrow \infty} \phi^{(n)}\left(t_{0}\right)\right)=\lim _{n \rightarrow \infty} \phi^{(n+1)}\left(t_{0}\right)=a .
$$

But this contradicts the fact that $\phi(a)<a$, since $a>0$, and completes the proof.

Lemma 2.1 shows that Browder's fixed point theorem and Matkowski's fixed point theorem are essentially equivalent. The advantage of Lemma 2.1 is that it allows us to check condition (1) for a given nondecreasing function $\phi$ more easily, provided we know that $\phi$ is right-continuous. For example, if $\phi_{1}, \phi_{2}:[0, \infty) \rightarrow[0, \infty)$ are two nondecreasing right-continuous functions satisfying (1), the function $\phi:=\max \left\{\phi_{1}, \phi_{2}\right\}$ satisfies (1) as well. In fact, $\phi_{1}(t)<t$ and $\phi_{2}(t)<t$ implies that also $\phi(t)<t$ for all $t>0$, and the assertion follows from Lemma 2.1. We will use this fact in the proof of our main result (Theorem 3.1 below).

We remark that the Browder-Matkowski fixed point theorem has found numerous applications; the interested reader is referred to the recent research monograph [9].

\section{MAIN RESULTS}

Before stating our main result on problem (1.4) we show that, as usual, every solution of (1.4) is a fixed point of an appropriate operator, and vice versa (see Lemma 3.1 below). Let $f, g:[a, b] \times \mathbb{R} \rightarrow \mathbb{R}$ be continuous functions. To simplify the notation we denote by

$$
F x(t)=f(t, x(t)), \quad G x(t)=g(t, x(t))
$$

the nonlinear superposition operators generated by $f$ and $g$, respectively. Moreover, we consider the composition

$$
\left(I_{\alpha} \circ F\right) x(t)=\frac{1}{\Gamma(\alpha)} \int_{a}^{t}(t-s)^{\alpha-1} f(s, x(s)) d s
$$

of the superposition operator $F$ with the linear fractional integral operator (1.5), as well as the composition

$$
(V \circ G) x(t)=\int_{a}^{t} g(s, x(s)) d s
$$

of the superposition operator $G$ with the linear Volterra operator

$$
V x(t)=\int_{a}^{t} x(s) d s
$$

In this notation, problem (1.4) may be written more concisely as operator equation

$$
D_{c}^{\alpha} x=F x+D_{c}^{\alpha-1} G x \quad(x \in C[a, b]) .
$$


Lemma 3.1. A function $x \in C[a, b]$ is a solution of the initial value problem (1.4) if and only if $x$ is a fixed point of the nonlinear operator $T$ defined by

$$
T x(t)=\theta_{1}+\left(\theta_{2}-g\left(a, \theta_{1}\right)\right)(t-a)+\left(I^{\alpha} \circ F\right) x(t)+(V \circ G) x(t) .
$$

Proof. Suppose that $x \in C[a, b]$ solves the initial value problem (1.4). Applying the operator $I^{\alpha}$ to both sides of (1.4) we obtain

$$
\left(I^{\alpha} \circ D_{c}^{\alpha}\right) x(t)=\left(I^{\alpha} \circ F\right) x(t)+\left(I^{\alpha} \circ D_{c}^{\alpha-1} \circ G\right) x(t)
$$

for any $t \in[a, b]$. Consequently,

$$
\begin{gathered}
x(t)-x(a)-x^{\prime}(a)(t-a)=\left(I^{\alpha} \circ F\right) x(t)+\left(I^{\alpha} \circ D_{c}^{\alpha-1} \circ G\right) x(t) \\
=\left(I^{\alpha} \circ F\right) x(t)+\left(I^{1} \circ I^{\alpha-1} \circ D_{c}^{\alpha-1} \circ G\right) x(t)=\left(I^{\alpha} \circ F\right) x(t)+\left(I^{1} \circ G\right) x(t)-\left(I^{1} \circ G\right) x(a) \\
=\left(I^{\alpha} \circ F\right) x(t)+(V \circ G) x(t)-g(a, x(a))(t-a),
\end{gathered}
$$

where we used (1.9) in the first, (1.6) in the second, and (1.8) in the third equality sign. Taking into account the initial conditions in (1.4), we end up with

$$
\begin{gathered}
x(t)=x(a)+x^{\prime}(a)(t-a)+\left(I^{\alpha} \circ F\right) x(t)+(V \circ G) x(t)-g(a, x(a))(t-a) \\
=\theta_{1}+\theta_{2}(t-a)+\left(I^{\alpha} \circ F\right) x(t)+(V \circ G) x(t)-g\left(a, \theta_{1}\right)(t-a)=T x(t),
\end{gathered}
$$

which shows that $x$ is a fixed point of the operator (3.3). Assume now that $x$ is a fixed point of the operator (3.3), i.e.,

$$
x(t)=\theta_{1}+\theta_{2}(t-a)+\left(I^{\alpha} \circ F\right) x(t)+(V \circ G) x(t)-g\left(a, \theta_{1}\right)(t-a) .
$$

Applying then the operator $D_{c}^{\alpha}$ to both sides of this equality yields

$$
\begin{gathered}
D_{c}^{\alpha} x(t)=D_{c}^{\alpha}\left(\theta_{1}\right)+\left(\theta_{2}-g\left(a, \theta_{1}\right) D_{c}^{\alpha}(t-a)+\left(D_{c}^{\alpha} \circ I^{\alpha} \circ F\right) x(t)+\left(D_{c}^{\alpha} \circ V \circ G\right) x(t)\right. \\
=F x(t)+\left(D_{c}^{\alpha-1} \circ G\right) x(t)=f(t, x(t))+D_{c}^{\alpha-1} g(t, x(t)),
\end{gathered}
$$

since $D_{c}^{\alpha} \circ V=D_{c}^{\alpha-1}$. Consequently, $x$ is a solution of (1.4).

We are now ready to prove a local existence and uniqueness result for continuous solutions of the initial value problem (1.4).

Theorem 3.1. Suppose that $f, g:[a, b] \times \mathbb{R} \rightarrow \mathbb{R}$ are continuous and satisfy the generalized Lipschitz conditions

$$
|f(t, x)-f(t, y)| \leq \phi_{f}(|x-y|)
$$

and

$$
|g(t, x)-g(t, y)| \leq \phi_{g}(|x-y|)
$$

for $t \in[a, b]$ and $x, y \in \mathbb{R}$, where $\phi_{f}$ and $\phi_{g}$ are comparison functions. Let $1<\alpha<2$. Moreover, assume that

$$
b-a+\frac{(b-a)^{\alpha}}{\Gamma(\alpha+1)} \leq 1 .
$$

Then problem (1.4) has a unique continuous solution on $[a, b]$. 
Proof. Consider the complete metric space $X=C[a, b]$ of real continuous functions on $[a, b]$, equipped with the usual metric $d(f, g)=\max \{|f(t)-g(t)|: t \in[a, b]\}$. We claim that the operator (3.3) maps the space $X$ into itself and satisfies, under the hypothesis (3.6), the generalized contraction condition (2.1) for a suitable $\phi$; the assertion follows then from Lemma 3.1.

Since $f$ and $g$ are continuous and $1<\alpha<2$, it is clear that, for $x \in X$, the function $T x$ given by (3.3) is continuous, which shows that $T(X) \subseteq X$. Now we verify (2.1) for $\phi:=\max \left\{\phi_{f}, \phi_{g}\right\}$. To this end, we estimate $d(T x, T y)$ as follows. For $t \in[a, b]$, we have

$$
\begin{gathered}
|T x(t)-T y(t)|=\left|(V \circ G) x(t)+\left(I^{\alpha} \circ F\right) x(t)-(V \circ G) y(t)-\left(I^{\alpha} \circ F\right) y(t)\right| \\
\leq \int_{a}^{t}|g(s, x(s))-g(s, y(s))| d s+\frac{1}{\Gamma(\alpha)} \int_{a}^{t}(t-s)^{\alpha-1}|f(s, x(s))-f(s, y(s))| d s \\
\leq \int_{a}^{t} \phi_{g}(|x(s)-y(s)|) d s+\frac{1}{\Gamma(\alpha)} \int_{a}^{t}(t-s)^{\alpha-1} \phi_{f}(|x(s)-y(s)|) d s \\
\leq(t-a) \phi_{g}(d(x, y))+\frac{\phi_{f}(d(x, y))}{\Gamma(\alpha)} \int_{a}^{t}(t-s)^{\alpha-1} d s \\
=(t-a) \phi_{g}(d(x, y))+\frac{(t-a)^{\alpha}}{\alpha \Gamma(\alpha)} \phi_{f}(d(x, y)) \leq\left[(b-a)+\frac{(b-a)^{\alpha}}{\Gamma(\alpha+1)}\right] \phi(d(x, y)) .
\end{gathered}
$$

Taking now the maximum over $t \in[a, b]$, the assertion follows from (3.6), and so we are done.

The condition (3.6) on the existence interval is of course rather restrictive. A considerably milder restriction is necessary if we suppose that $g(t, u) \equiv 0$ in (1.4), i.e., we consider the initial value problem

$$
\left\{\begin{array}{l}
D_{c}^{\alpha} x(t)=f(t, x(t)), \quad(a<t<b), \\
x(a)=\theta_{1}, x^{\prime}(a)=\theta_{2} .
\end{array}\right.
$$

Here we get the following existence and uniqueness result which is parallel to Theorem 3.1.

Theorem 3.2. Suppose that $f:[a, b] \times \mathbb{R} \rightarrow \mathbb{R}$ is continuous and satisfies the generalized Lipschitz conditions (3.4) for $t \in[a, b]$ and $x, y \in \mathbb{R}$, where $\phi_{f}$ has the property (1) or (2) stated in Lemma 2.1. Let $1<\alpha<2$. Moreover, assume that

$$
b-a \leq \Gamma(\alpha+1)^{\frac{1}{\alpha}} .
$$

Then problem (3.7) has a unique continuous solution on $[a, b]$.

Proof. The proof is similar to that of Theorem 3.1, so we only sketch the differences. We replace the operator (3.3) by the simpler operator

$$
T x(t)=\theta_{1}+\theta_{2}(t-a)+\left(I^{\alpha} \circ F\right) x(t) .
$$

A similar calculation as before leads then to the estimate

$$
d(T x, T y) \leq \frac{(b-a)^{\alpha}}{\alpha \Gamma(\alpha)} \phi_{f}(d(x, y))=\frac{(b-a)^{\alpha}}{\Gamma(\alpha+1)} \phi_{f}(d(x, y)),
$$

and the statement follows. 


\section{AN EXAMPLE}

We illustrate our abstract results by means of two simple examples. To this end, observe that the function $\phi:[0, \infty) \rightarrow[0, \infty)$ defined by $\phi(t)=\arctan t$ is continuous and nondecreasing, and satisfies condition (2.3), because $\phi(0)=0$ and $0<\phi^{\prime}(t)<1$ for $t>0$. By Lemma 2.1, it also satisfies condition (2.2). We use this comparison function in the following.

Example 4.1. Consider the fractional initial value problem

$$
\left\{\begin{array}{l}
D_{c}^{3 / 2} x(t)=\arctan (t+|x(t)|), \quad(a<t<b), \\
x(a)=\theta_{1}, x^{\prime}(a)=\theta_{2},
\end{array}\right.
$$

where $\theta_{1}, \theta_{2} \in \mathbb{R}$ as before. Since the function $t \mapsto \arctan t$ is strictly increasing and concave on $[0, \infty)$, the function $f(t, u)=\arctan (t+|u|)$ satisfies

$$
|f(t, x)-f(t, y)| \leq \arctan (|| x|-| y||) \leq \arctan (|x-y|),
$$

which is nothing else but (3.4) with $\phi_{f}(t)=\arctan t$. So if we choose, according to (3.8),

$$
b-a \leq \Gamma(5 / 2)^{2 / 3} \approx 1.209,
$$

from Theorem 3.2 we conclude that the initial value problem (4.1) has a unique continuous solution on $[a, b]$.

The next example is a slight generalization of Example 4.1, inasmuch as it illustrates Theorem 3.1.

Example 4.2. Consider the fractional initial value problem

$$
\left\{\begin{array}{l}
D_{c}^{3 / 2} x(t)=\arctan (t+|x(t)|)+D_{c}^{1 / 2} \log (1+|x(t)|), \quad(a<t<b), \\
x(a)=\theta_{1}, x^{\prime}(a)=\theta_{2},
\end{array}\right.
$$

where $\theta_{1}, \theta_{2} \in \mathbb{R}$ as before. Here we have to consider, in addition to the function $\phi_{f}(t)=\arctan t$, the comparison function $\phi_{g}(t)=\log (1+t)$ for $t>0$. But this has essentially the same properties as $\phi_{f}$. So if we choose, according to (3.6),

$$
b-a+\frac{(b-a)^{3 / 2}}{\Gamma(5 / 2)} \leq 1
$$

from Theorem 3.1 we conclude that the initial value problem (4.2) has a unique continuous solution on $[a, b]$.

\section{CONCLUDING REMARKS}

Theorem 3.1 and Theorem 3.2 give existence and uniqueness for continuous solutions of the fractional initial value problem (1.4). However, the space $C[a, b]$ is not very suitable for fractional integral and differential operators. Instead, such operators are "better behaved" in Lebesgue, Sobolev, or Hölder spaces. For example, it is well-known that the linear operator (1.5) is bounded between $L_{p}[a, b]$ and $L_{p /(1-p \alpha)}[a, b]$ for $1<p<1 / \alpha$, or between $L_{p}[a, b]$ and $C^{\alpha-1 / p}[a, b]$, or between $C^{\beta}[a, b]$ and $C^{\beta-\alpha}[a, b]$ for $0<\beta<1-\alpha<1$. Here $C^{\beta}[a, b]$ denotes the Banach space of all Hölder continuous (with exponent $\beta \in(0,1])$ functions $x:[a, b] \rightarrow \mathbb{R}$, equipped with the natural norm

$$
\|x\|_{\beta}=|x(a)|+\sup \left\{\frac{|x(s)-x(t)|}{|s-t|^{\beta}}: a \leq s, t \leq b, s \neq t\right\} .
$$


However, if we try to imitate our previous arguments by imposing the generalized contraction condition (2.1) on the superposition operators (3.1), we encounter a very unpleasant surprise: such a contraction condition can be satisfied only if the underlying functions $f$ and $g$ are affine. As far as we know, a degeneracy phenomenon of this kind has been proved first for the classical contraction condition by Matkowski [7] in the space of Lipschitz continuous functions, and subsequently in many other function spaces. Although the proof in our case is quite similar, we state it here because it gives some insight into the problem.

Theorem 5.1. Suppose that the operator F from (3.1) satisfies a generalized contraction condition of type (2.1) in the Hölder space $C^{\beta}[a, b]$, i.e.,

$$
\|F x-F y\|_{\beta} \leq \phi\left(\|x-y\|_{\beta}\right)
$$

for some comparison function $\phi$. Then the function $f$ is affine, i.e., there exist $A, B \in C^{\beta}[a, b]$ such that $f(t, u)=A(t) u+B(t)$.

Proof. Without loss of generality, we take $[a, b]=[0,1]$. For $0<\sigma<\tau<1$ and $\xi, \eta \in \mathbb{R}$, we define two functions $x, y:[0,1] \rightarrow \mathbb{R}$ by

$$
x(t)= \begin{cases}\eta, & \text { for } \quad 0 \leq t<\sigma, \\ \frac{\xi(t-\sigma)}{\tau-\sigma}+\eta, & \text { for } \quad \sigma \leq t \leq \tau, \\ \xi+\eta, & \text { for } \tau<t \leq 1,\end{cases}
$$

and $y(t)=x(t)-\eta$. A straightforward calculation shows that $\|x-y\|_{\beta}=|\eta|$ and

$$
\|F x-F y\|_{\beta}=|f(0, \eta)-f(0,0)|+\frac{|f(\tau, \xi+\eta)-f(\tau, \xi)-f(\sigma, \eta)+f(\sigma, 0)|}{(\tau-\sigma)^{\beta}} .
$$

Inserting this into the contraction condition (5.1) yields

$$
|f(0, \eta)-f(0,0)|+\frac{|f(\tau, \xi+\eta)-f(\tau, \xi)-f(\sigma, \eta)+f(\sigma, 0)|}{(\tau-\sigma)^{\beta}} \leq \phi(|\eta|) .
$$

Now, multiplying both sides of (5.2) by $(\tau-\sigma)^{\beta}$ and letting $\tau \rightarrow \sigma$, we obtain

$$
f(\sigma, \xi+\eta)-f(\sigma, \xi)-f(\sigma, \eta)+f(\sigma, 0)=0 .
$$

But this means that the function $\ell_{\sigma}(u):=f(\sigma, u)-f(\sigma, 0)$ is additive and continuous, so $\ell_{\sigma}(u)=A(\sigma) u$. Moreover, if we put $f(\sigma, 0)=B(\sigma)$ we get the representation $f(\sigma, u)=A(\sigma) u+B(\sigma)$ as claimed.

Theorem 5.1 is of course quite disappointing: it shows that we may apply generalized contraction conditions like (5.1) only if our problem is actually linear! So it seems natural to apply other fixed point theorems. Here the classical Schauder theorem and its various generalizations are a good choice, because compactness criteria are known in many function spaces. However, we have to pay a price for this: the application of topological fixed point theorems requires the existence of an invariant convex, closed and bounded set, e.g., a closed ball. To derive the necessary growth estimates for the operator (3.3), one may use then the fact that the Riemann-Liouville operator (1.5) and the Volterra operator (3.2) have spectral radius zero. Details will be given in a subsequent paper.

\section{Acknowledgements}


The second and third authors were partially supported by the projects MTM2016-79436-P and ULPGC 2014-04. The first author gratefully acknowledges hospitality of the Universidad de Las Palmas de Gran Canaria.

\section{REFERENCES}

[1] A. Aghajani, J. Banaś, N. Sabzali, Some generalizations of Darbo fixed point theorem and applications, Bull. Belg. Math. Soc. Simon Stevin 20 (2013), 345-358.

[2] F. Browder, On the convergence of successive approximations for nonlinear functional equations, Indag. Math. 30 (1968), 27-35.

[3] R.A.C. Ferreira, Existence and uniqueness of solutions for two-point fractional boundary value problems, Electron. J. Differential Equations 202 (2016), 1-5.

[4] W.G. Kelley, A.C. Peterson The Theory of Differential Equations, Second edition, Universitext, Springer, New York, 2010.

[5] A.A. Kilbas, H.M. Srivastava, J.J. Trujillo, Theory and Applications of Fractional Differential Equations, Elsevier, Amsterdam 2006.

[6] J. Matkowski, Integrable solutions of functional equations, Dissertationes Math. 127 (1975), 1-68.

[7] J. Matkowski, Functional equations and Nemytskij operators, Funkc. Ekvacioj 25 (1982), 127-132.

[8] I. Podlubny, Fractional Differential Equations, Academic Press, San Diego 1999.

[9] S. Reich, A.J. Zaslavski, Genericity in Nonlinear Analysis, Springer, New York 2014.

[10] I. Rus, A. Petrusel, G. Petrusel, Fixed Point Theory, Cluj University Press, Cluj, 2008. 